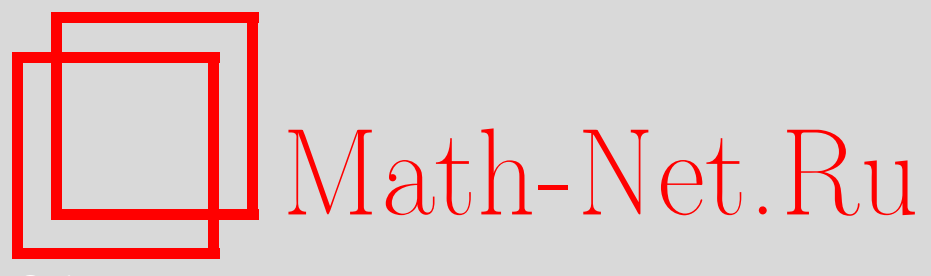

А. С. Воронин, Н. Б. Медведева, Асимптотика преобразования монодромии в некоторых классах монодромных ростков, Изв. РАН. Сер. матем., 2013, том 77, выпуск $2,35-52$

DOI: https://doi.org/10.4213/im7984

Использование Общероссийского математического портала Math-Net.Ru подразумевает, что вы прочитали и согласны с пользовательским соглашением http://www.mathnet.ru/rus/agreement

Параметры загрузки:

IP: 35.174 .16 .151

26 апреля 2023 г., 18:21:43

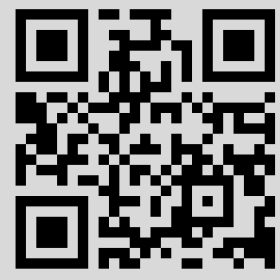


УДК 517.9

\author{
А. С. Воронин, Н. Б. Медведева
}

\title{
Асимптотика преобразования монодромии в некоторых классах монодромных ростков
}

\begin{abstract}
Вычисляется второй член асимптотики преобразования монодромии монодромной особой точки аналитического векторного поля на плоскости, диаграмма Ньютона которого состоит из одного или двух ребер. В рассматриваемых случаях главный член преобразования монодромии совпадает с тождественной функцией. Случай с двумя ребрами характеризуется тем, что в результате раздутия особенности по диаграмме Ньютона возникает особая точка, являющаяся вырожденным седлом. Полученные результаты позволяют сформулировать достаточное условие наличия фокуса и построить границу устойчивости в рассматриваемых классах векторных полей.
\end{abstract}

Библиография: 13 наименований.

Ключевые слова: монодромная особая точка, раздутие особенностей, фокус, центр, преобразование монодромии.

DOI: $10.4213 / \operatorname{im} 7984$

\section{Введение}

ОПРЕДЕЛЕНИЕ 0.1 [1]. Особая точка векторного поля называется монодромной, если существуют окрестность этой точки и дуга с вершиной в этой точке, гладкая и трансверсальная полю всюду вне вершины (полутрансверсаль), такие, что векторное поле в окрестности с выброшенной дугой топологически орбитально эквивалентно стандартному векторному полю (рис. 1). Точнее, существует непрерывное отображение замкнутого прямоугольника на замыкание окрестности особой точки, гомеоморфно переводящее внутренность прямоугольника на дополнение окрестности до упомянутой полутрансверсали и преобразующее горизонтальные прямые в траектории исходного векторного поля; вертикальные стороны оно отображает на полутрансверсаль, а нижнюю горизонтальную сторону переводит в особую точку. Каждая траектория исходного поля с началом на полутрансверсали, достаточно близким к особой точке, сделав один виток вблизи этой точки, возвращается на ту же полутрансверсаль. Отображение, переводящее начальную точку каждой такой дуги траектории в ее конец (точку первого возвращения на полутрансверсаль), называется преобразованием монодромии особой точки (см. рис. 1).

Во внутренних точках полутрансверсали преобразование монодромии имеет тот же класс гладкости, что и векторное поле, и аналитично вместе с ним. Однако оно может не продолжаться гладко в вершину полутрансверсали даже в случае аналитического векторного поля.

Работа выполнена при частичной финансовой поддержке РФФИ (грант № 10-01-00587-а) и ФЦП (грант № 02.740.110612).

(C) А. С. Воронин, Н. Б. МедведевА, 2013 


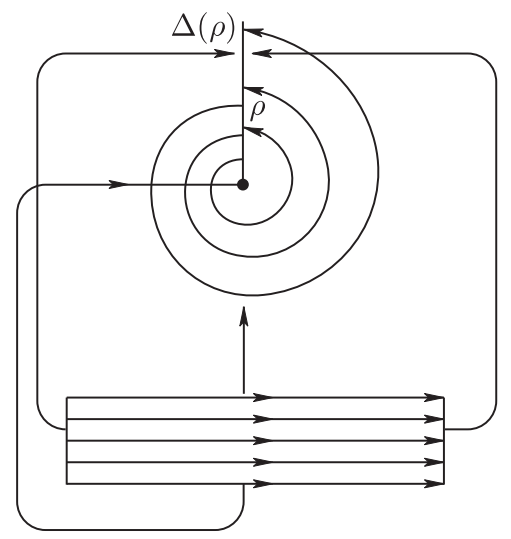

Рис. 1. Преобразование монодромии

Из определения 0.1 следует, что каждая траектория в окрестности монодромной особой точки является или спиралью, или окружностью.

Аналогично определяются монодромный сложный цикл и преобразование монодромии сложного цикла - объединения конечного числа особых точек и неодноточечных траекторий, входящих в эти особые точки при $t \rightarrow \pm \infty[2]$. Замкнутый прямоугольник непрерывно отображается на замыкание полуокрестности сложного цикла, как в случае монодромной особой точки.

Подобно преобразованию монодромии определяется отображение соответствия одной полутрансверсали с вершиной на сложном цикле в другую полутрансверсаль. Образ и прообраз точки при отображении соответствия лежат на одной траектории. Преобразование монодромии сложного цикла может быть разбито в суперпозицию отображений соответствия. Сложные циклы возникают, например, из особых точек при раздутии особенностей.

Пусть $\rho$ - параметр на полутрансверсали, $\Delta(\rho)$ - преобразование монодромии. Если $\Delta(\rho) \equiv \rho$, то особая точка является центром. Доказано [1], [3], что при подходящем выборе полутрансверсали выполнено $\Delta(\rho)=C_{1} \rho+o(\rho)$ при $\rho \rightarrow 0, C_{1}>0$. Неравенство $\ln C_{1} \neq 0$ является достаточным условием того, чтобы особая точка была фокусом.

В работе [4] вычислена величина $\ln C_{1}$ для монодромной особой точки произвольного аналитического векторного поля. Ответ формулируется в терминах диаграмм Ньютона векторных полей, возникающих в процессе раздутия особенностей.

В [5] показано, что если все ребра диаграммы Ньютона $\Gamma$ четные, то $\ln C_{1} \equiv 0$ на всем пространстве Г-невырожденных векторных полей (определение дано в работе [5]) с монодромной особой точкой, т. е. преобразование монодромии имеет асимптотику $\Delta(\rho)=\rho+o(\rho)$. Таким образом, чтобы получить достаточное условие фокуса и построить границу устойчивости в этом пространстве, необходимо вычислять второй член асимптотики. 
В статьях [6] и [7] вычисляется второй член асимптотики преобразования монодромии Г-невырожденного векторного поля в случае диаграммы Ньютона, состоящей из двух четных ребер.

В настоящей работе построены два члена асимптотики преобразования монодромии векторного поля с монодромной особой точкой, имеющего диаграмму Ньютона, которая состоит из одного четного ребра, и принадлежащего немного более широкому классу, чем класс Г-невырожденных векторных полей (теорема 0.1).

В работах [8] и [9] предлагаются алгоритмы вычисления коэффициентов асимптотического разложения преобразования монодромии с помощью методов раздутия особенностей, специфических для случая одного ребра диаграммы Ньютона. В настоящей статье преобразование монодромии исследуется с помощью другого метода раздутия, связанного с диаграммами Ньютона, который является удобным для исследования монодромных особых точек с любыми диаграммами Ньютона и любыми вырождениями. Г-невырожденный случай диаграммы с двумя ребрами ранее был полностью исследован в [6] и [7] с помощью упомянутого метода. Формула теоремы 0.1 заполняет образовавшийся пробел в исследовании, в дальнейшем предполагается ее применить к исследованию вопроса об аналитической разрешимости проблемы устойчивости на плоскости.

Также в настоящей работе вычислен второй член асимптотики преобразования монодромии векторного поля, имеющего диаграмму Ньютона, состоящую из двух ребер, причем условия Г-невырожденности нарушаются таким образом, что в результате раздутия по диаграмме Ньютона появляется особая точка типа вырожденного седла (в Г-невырожденном случае аналогичная особая точка является невырожденным седлом). Второй член асимптотики преобразования монодромии в случае с вырожденным седлом вычисляется впервые.

Случай диаграммы с двумя ребрами (при наличии, конечно, условий невырожденности на ребрах) является наиболее простым случаем, в котором возникают особые точки на вклеенной кривой после раздутия. Построенные в [6] и [7], а также в данной статье асимптотики отображений соответствия для седловых особых точек позволят вычислить два члена асимптотики преобразования монодромии в любом как угодно вырожденном случае.

Для того чтобы сформулировать результаты, приведем некоторые определения, связанные с диаграммой Ньютона.

Рассмотрим аналитическое векторное поле (росток векторного поля) в окрестности точки нуль на плоскости, которое определяет динамическую систему

$$
\dot{x}=X(x, y), \quad \dot{y}=Y(x, y) .
$$

ОПРЕДЕЛЕНИЕ 0.2. Рассмотрим тейлоровские разложения

$$
y X(x, y)=\sum_{i+j=1}^{\infty} a_{i j} x^{i} y^{j}, \quad x Y(x, y)=\sum_{i+j=1}^{\infty} b_{i j} x^{i} y^{j} .
$$

Носителем системы $(0.1)$, а также соответствующего ей векторного поля называется множество таких пар $(i, j)$, что $\left(a_{i j}, b_{i j}\right) \neq(0,0)$. Вектор $\left(a_{i j}, b_{i j}\right)$ называется векторным коэффициентом точки носителя $(i, j)$. 
ОПРЕДЕЛЕНИЕ 0.3. Рассмотрим множество $\bigcup_{(i, j)}\left\{(i, j)+\mathbb{R}_{+}^{2}\right\}$, где $\mathbb{R}_{+}^{2}$ - положительный квадрант; объединение берется по всем точкам $(i, j)$, принадлежащим носителю. Граница выпуклой оболочки этого множества состоит из двух открытых лучей и ломаной, которая может состоять и из одной точки. Эта ломаная называется диаграммой Нъютона системы (0.1), а также соответствующего ей векторного поля. Звенья ломаной называются ребрами диаграммы Ньютона, а их концы - вершинами диаграммы Ньютона.

ОПРЕДЕЛЕНИЕ 0.4. Показателем ребра диаграммы Ньютона называется положительное рациональное число, равное тангенсу угла между ребром и осью ординат.

ОПРЕДЕЛЕНИЕ 0.5. Пусть $\alpha=m / n-$ несократимая дробь. Ребро диаграммы Ньютона с показателем $\alpha$ назовем четным, если одно из чисел $m$ и $n$ четно, и нечетным в противном случае.

ОПРЕДЕЛЕНИЕ 0.6. Рассмотрим ребро $\ell$ диаграммы Ньютона системы (0.1) с показателем $\alpha=m / n$, где $m / n$ - несократимая дробь. Члены разложения (0.2) сгруппируем таким образом, что

$$
y X(x, y)=\sum_{k=0}^{\infty} X_{k}(x, y), \quad x Y(x, y)=\sum_{k=0}^{\infty} Y_{k}(x, y)
$$

где

$$
X_{k}(x, y)=\sum_{n i+m j=k+k_{0}} a_{i j} x^{i} y^{j}, \quad Y_{k}(x, y)=\sum_{n i+m j=k+k_{0}} b_{i j} x^{i} y^{j}
$$

- квазиоднородные полиномы степени $k+k_{0}$ с весами $n$ и $m$ переменных $x$ и $y$ соответственно, $k_{0}>0$. Обозначим $F_{k}(x, y)=n Y_{k}(x, y)-m X_{k}(x, y)$. Положим

$$
\Phi_{0}=\frac{X_{0}}{F_{0}}, \quad \Psi_{0}=-\frac{Y_{0}}{F_{0}} ; \quad \Phi_{1}=\frac{F_{0} X_{1}-F_{1} X_{0}}{F_{0}^{2}}, \quad \Psi_{1}=\frac{F_{1} Y_{0}-F_{0} Y_{1}}{F_{0}^{2}}=-\alpha \Phi_{1} .
$$

ПреДЛОЖЕНИЕ 0.1 [5]. Пустъ $m / n$ - несократимая дробъ. Для любого квазиоднородного полинома $R(x, y)$ с весами $n$ и m переменных $x$ и у справедливо разложение

$$
R(x, y)=A x^{s_{1}} y^{s_{2}} \prod_{i}\left(y^{n}-b_{i} x^{m}\right)^{k_{i}},
$$

где $b_{i}$ - различные ненулевые комплексные числа, $k_{i} \geqslant 0, s_{1} \geqslant 0, s_{2} \geqslant 0$.

Многочлен вида $y^{n}-b_{i} x^{m}, b_{i} \neq 0$, называется простым делителем полинома $R(x, y)$.

ТЕОрема 0.1. Пусть векторное поле $V$ имеет монодромную особую точку $(0,0)$, и пусть его диаграмма Нъютона Г состоит из одного четного ребра c показателем $\mathrm{m} / \mathrm{n}$ и имеет по одной вериине на каждой координатной оси, причем квазиоднородный многочлен $F_{0}(x, y)$, соответствующий ребру диаграммы Нъютона Г, не имеет вещественных простых делителей. В предположении, что т четно, преобразование монодромии особой точки $(0,0)$ векторного поля $V$, определенное вблизи нуля на положительной полуоси у с параметром $\rho=y^{1 / m}$, имеет при $\rho \rightarrow 0$ асимптотику вида $\hat{\Delta}(\rho)=\rho\left(1+C_{2} \rho+o(\rho)\right)$, 
причем уравнение $C_{2}=0$ эквивалентно уравнению

$$
\int_{-\infty}^{+\infty} \frac{\Phi_{1}(1, \xi)}{\xi} \exp \left(\int_{0}^{\xi} \frac{\Phi_{0}(1, \tau)}{\tau} d \tau\right) d \xi=0 .
$$

Случай нечетного $m$ получается из рассмотренного в теореме 0.1 заменой переменных $x$ на $y$ и обратно.

ЗАмЕчАниЕ 0.1. При смене трансверсали, параметра на трансверсали и обращении времени коэффициент $C_{2}$ умножается на ненулевую величину. В формулировке теоремы 0.1 мы не выписываем сам коэффициент при втором члене асимптотики, а только уравнение $C_{2}=0$, во-первых, в силу его громоздкости, во-вторых, потому, что для дальнейших исследований нам нужно уметь выписывать уравнение границы устойчивости, т. е. $C_{2}=0$. Из доказательства теоремы 0.1 можно извлечь и формулу для $C_{2}$. При указанном выборе трансверсали если траектории векторного поля $V$ движутся по направлению часовой стрелки, то $C_{2}=-c_{0} I$, где $I-$ левая часть уравнения $(0.4)$, $c_{0}=\exp \left(\int_{1}^{0} \frac{\Phi_{0}(1, w)}{w} d w\right) \exp \left(\int_{+\infty}^{1}\left(\Phi_{0}(1, w)+\frac{1}{m}\right) \frac{d w}{w}\right)$.

ПРЕДЛОЖЕНИЕ 0.2. Существуют такие наборы коэффициентов многочленов $X_{1}$ и $Y_{1}$, что при любых коэффициентах многочленов $X_{0}$ и $Y_{0}$, удовлетворяющих условиям теоремы 0.1 , левая часть уравнения (0.4) не равна нулю.

Из предложения 0.2 следует, что $C_{2} \not \equiv 0$, а значит, уравнение $C_{2}=0$ задает границу устойчивости в рассматриваемом классе.

Пусть векторное поле $V$ имеет монодромную особую точку $(0,0)$, и пусть его диаграмма Ньютона состоит из двух ребер $\ell$ и $\tilde{\ell}$ с показателями $\alpha=m / n$ и $\tilde{\alpha}=\tilde{m} / \tilde{n}$ (несократимые дроби), $\alpha<\tilde{\alpha}$, и имеет по одной вершине на каждой координатной оси. Пусть $\Psi_{0}, \Psi_{1}$ определяются формулами $(0.3)$ и соответствуют ребру $\ell$.

Условия Г-невырожденности включают неравенства $n b-m a \neq 0, \tilde{n} b-\tilde{m} a \neq 0$, где $(a, b)$ - векторный коэффициент вершины, соединяющей ребра $\ell$ и $\tilde{\ell}$ диаграммы Ньютона Г. В настоящей статье мы предполагаем, что одна из величин $n b-m a, \tilde{n} b-\widetilde{m} a$ обращается в нуль. Это означает, что в результате раздутия по диаграмме Ньютона возникает особая точка, являющаяся вырожденным седлом.

Теорема 0.2. Пусть векторное поле $V$ имеет монодромную особую точку $(0,0)$, и пусть его диаграмма Нъютона состоит из двух ребер $\ell$ u $\tilde{\ell}$ с показателями $\alpha=m / n$ и $\tilde{\alpha}=\widetilde{m} / \tilde{n}$ (несократимые дроби), $\alpha<\tilde{\alpha}$, и имеет по одной вершине на каждой координатной оси. Пусть, кроме того, выполняются условия:

1) квазиоднородные многочлены $F_{0}(x, y)$ и $\widetilde{F}_{0}(x, y)$, соответствующие ребрам $\ell$ и $\tilde{\ell}$, не имеют вещественных простых делителей;

2) $\tilde{n} b-\widetilde{m} a=0$, где $(a, b)$ - векторный коэффициент вериины, соединяющей ребра $\ell$ u $\tilde{\ell}$

3) ребро $\ell$ четно;

4) траектории векторного поля $V$ движутся вокруг нуля по направлению часовой стрелки. 
Тогда преобразование монодромии векторного поля $V$, определенное вблизи нуля на положительной полуоси у с параметром $\rho=y^{1 / m}$, имеет асимптотику вида

$$
\hat{\Delta}(\rho)=\rho\left(1+C_{2} \rho+o(\rho)\right), \quad \rho \rightarrow 0,
$$

где в случае, когда $m$ нечетно,

$$
C_{2}=2 \int_{-\infty}^{+\infty} \frac{\Psi_{1}(\xi, 1)}{\xi} \exp \left(\int_{0}^{\xi} \frac{\Psi_{0}(\tau, 1)}{\tau} d \tau\right) d \xi
$$

а в случае, когда $m$ четно,

$$
\begin{array}{r}
C_{2}=\int_{-\infty}^{+\infty} \frac{\Psi_{1}(\xi, 1)}{\xi} \exp \left(\int_{0}^{\xi} \frac{\Psi_{0}(\tau, 1)}{\tau} d \tau\right) d \xi-\exp \left(\int_{0}^{+\infty}\left(\Psi_{0}(\xi, 1)-\Psi_{0}(\xi,-1)\right) \frac{d \xi}{\xi}\right) \\
\times \int_{-\infty}^{+\infty} \frac{\Psi_{1}(\xi,-1)}{\xi} \exp \left(\int_{0}^{\xi} \frac{\Psi_{0}(\tau,-1)}{\tau} d \tau\right) d \xi .
\end{array}
$$

Случай, когда $n b-m a=0$ и $\tilde{\ell}$ четно, получается из рассматриваемого в теореме 0.2 заменой переменных $x \leftrightarrow y$. В случае нечетного $\ell$ имеем $\ln C_{1} \not \equiv 0$ [4].

Через $\mid \overline{\int_{a}^{b} f(\xi) d \xi}$ обозначается интеграл Адамара [10] от функции $f(x)$ (конечная часть несобственного интеграла), $-\infty \leqslant a<b \leqslant+\infty$.

СлеДСтвиЕ 0.1. В случае, когда $m$ четно, условие $C_{2}=0$ может быть записано в виде

$$
\begin{aligned}
\int_{-\infty}^{+\infty} \frac{\Psi_{1}(\xi, 1)}{\xi} \exp \left(\mid \overline{\int_{+\infty}^{\xi}} \frac{\Psi_{0}(\tau, 1)}{\tau} d \tau\right) d \xi \\
\quad=\int_{-\infty}^{+\infty} \frac{\Psi_{1}(\xi,-1)}{\xi} \exp \left(\mid \overline{\int_{+\infty}^{\xi} \frac{\Psi_{0}(\tau,-1)}{\tau} d \tau}\right) d \xi .
\end{aligned}
$$

ПРЕДЛОЖЕНИЕ 0.3. Существуют такие наборы коэфбиииентов многочленов $X_{1}$ и $Y_{1}$, что при любых коэффициентах многочленов $X_{0}$ и $Y_{0}$, удовлетворяющих условиям теоремы 0.2 , выполняется неравенство $C_{2} \neq 0$.

Из предложения 0.3 следует, что уравнение $C_{2}=0$ задает границу устойчивости в рассматриваемом классе.

Авторы благодарят Ю. С. Ильяшенко, С. М. Воронина, В. П. Варина за полезные замечания.

\section{§ 1. Случай одного ребра}

ДоКАЗАТЕЛЬСТво тЕОРЕмы 0.1. В случае диаграммы Ньютона, состоящей из одного ребра с показателем $\alpha=m / n$, метод раздутия по диаграмме Ньютона состоит в следующем. Окрестность нуля в первом квадранте плоскости $(x, y)$ делится на два сектора

$$
S_{0}=\left\{\frac{1}{\varepsilon} x^{\alpha} \leqslant y<\delta, x>0\right\}, \quad S_{1}=\left\{\frac{1}{\varepsilon} x^{\alpha} \geqslant y \geqslant 0,0 \leqslant x<\delta\right\},
$$

где $\delta, \varepsilon-$ малые положительные числа. 
В секторе $S_{0}$ делается замена переменных $x=\bar{z}^{n} \bar{w}, y=\bar{z}^{m}$, в результате чего он превращается в прямоугольник $P_{0}=\left\{0<\bar{w}<\varepsilon^{1 / \alpha}, 0<\bar{z}<\delta^{1 / m}\right\}$. Аналогично, в секторе $S_{1}$ делается замена $x=z^{n}, y=z^{m} w$, в результате чего он превращается в прямоугольник $P_{1}=\left\{0<w<1 / \varepsilon, 0<z<\delta^{1 / n}\right\}$ (рис. 2). Замены переменных сопровождаются делением на подходящие степени переменных. Прямоугольники $P_{0}$ и $P_{1}$ склеиваются с помощью функций перехода сторонами, которые соответствуют границе между секторами $S_{0}$ и $S_{1}$. Более подробно процесс раздутия описан в [11].

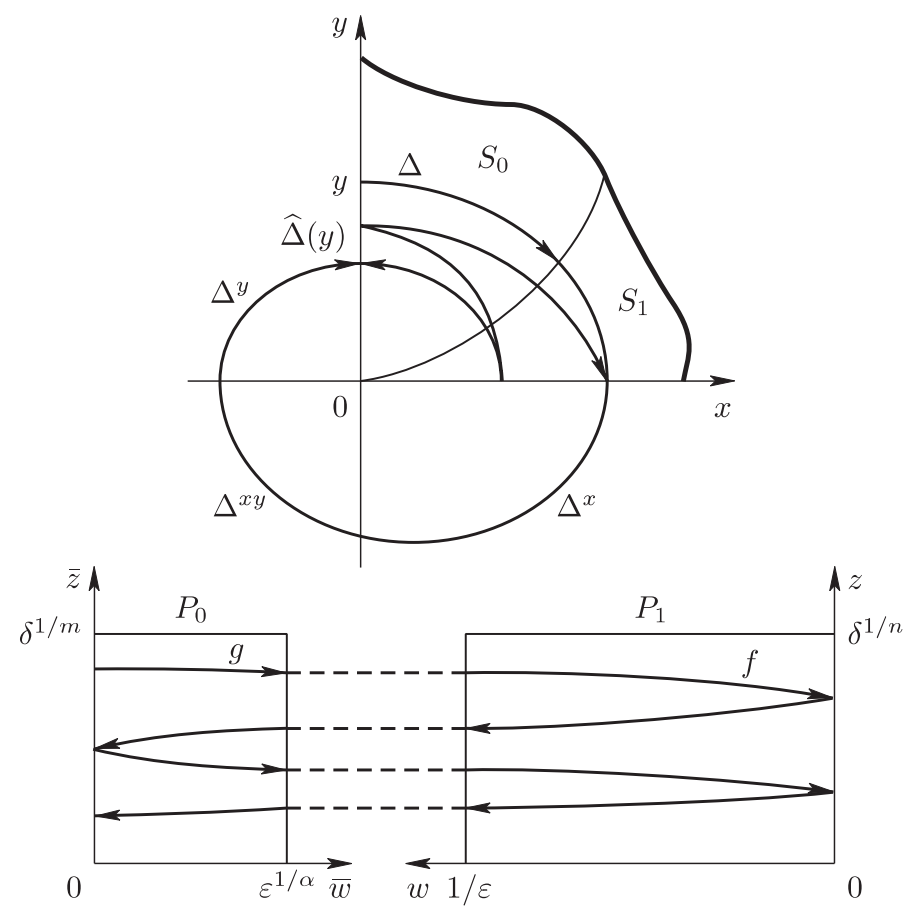

Рис. 2. Раздутие особенности в случае одного ребра

Пусть $S^{x}, S^{y}$ - отражения плоскости $(x, y)$ относительно осей $x$ и $y$ соответственно, $S^{x y}=S^{x} \circ S^{y}$. Образы векторного поля $V$ при отражениях $S^{x}, S^{y}$, $S^{x y}$ обозначим $V^{x}, V^{y}, V^{x y}$ соответственно. Рассмотрим в первом квадранте векторные поля $V, V^{x}, V^{y}, V^{x y}$ и применим к каждому из них описанный выше процесс раздутия особенности.

Из условия, что многочлен $F_{0}(x, y)$ не имеет вещественных простых делителей, и из того, что диаграмма Ньютона Г имеет по одной вершине на каждой координатной оси, следует, что векторные поля, полученные после раздутия векторных полей $V, V^{x}, V^{y}, V^{x y}$ и определенные в прямоугольниках $P_{0}, P_{1}$, не имеют особых точек на сторонах $\bar{z}=0$ (соответственно, $z=0$ ) прямоугольников $P_{0}$ (соответственно, $\left.P_{1}\right)[11]$.

Далее все обозначения, отвечающие отраженным векторным полям, будут такие же, как для векторного поля $V$, но с соответствующим индексом вверху. 
В [5, с. 170] доказано, что

$$
\Phi_{k}^{y}(x, y)=\Phi_{k}(-x, y), \quad \Phi_{k}^{x}(x, y)=\Phi_{k}(x,-y), \quad \Phi_{k}^{x y}(x, y)=\Phi_{k}(-x,-y) .
$$

Если $m$ нечетно, то

$$
\Phi_{0}(x,-y)=\Phi_{0}(x, y), \quad \Phi_{1}(x,-y)=-\Phi_{1}(x, y), \quad \Psi_{0}(x,-y)=\Psi_{0}(x, y) .
$$

Если $m$ четно, то

$$
\Phi_{0}(-x, y)=\Phi_{0}(x, y), \quad \Phi_{1}(-x, y)=-\Phi_{1}(x, y), \quad \Psi_{0}(-x, y)=\Psi_{0}(x, y) .
$$

Отображения соответствия $f:\{w=1 / \varepsilon\} \rightarrow\{w=0\}$ и $g:\{\bar{w}=0\} \rightarrow\{\bar{w}=$ $\left.\varepsilon^{1 / \alpha}\right\}$ в прямоугольниках $P_{1}$ и $P_{0}$ вдоль траекторий векторных полей, полученных после замен переменных, с учетом склейки задаются соответственно формулой [11]

$$
f(z)=a_{0} z\left(1+a_{1} z+o(z)\right)
$$

где

$$
a_{0}=\exp \left(\int_{1 / \varepsilon}^{0} \frac{\Phi_{0}(1, \xi)}{\xi} d \xi\right), \quad a_{1}=a_{0} \int_{1 / \varepsilon}^{0} \frac{\Phi_{1}(1, \xi)}{\xi} \exp \left(\int_{0}^{\xi} \frac{\Phi_{0}(1, \tau)}{\tau} d \tau\right) d \xi
$$

и формулой

$$
g(\bar{z})=b_{0} \varepsilon^{1 / m} \bar{z}\left(1+b_{1} \bar{z}+o(\bar{z})\right)
$$

где

$$
b_{0}=\exp \left(\int_{0}^{\varepsilon^{1 / \alpha}} \frac{\Psi_{0}(\xi, 1)}{\xi} d \xi\right), \quad b_{1}=\int_{0}^{\varepsilon^{1 / \alpha}} \frac{\Psi_{1}(\xi, 1)}{\xi} \exp \left(\int_{0}^{\xi} \frac{\Psi_{0}(\tau, 1)}{\tau} d \tau\right) d \xi .
$$

Пусть $\Delta$ - отображение соответствия для первого квадранта, определенное вблизи нуля на оси ординат с параметром $\bar{z}=y^{1 / m}$ (см. рис. 2). Тогда

$$
\Delta(\bar{z})=f \circ g(\bar{z})=c_{0} \bar{z}\left(1+c_{1} \bar{z}+o(\bar{z})\right), \quad \Delta^{-1}(z)=c_{0}^{-1} z\left(1-c_{1} c_{0}^{-1} z+o(z)\right),
$$

где $c_{0}=a_{0} b_{0} \varepsilon^{1 / m}, c_{1}=b_{1}+a_{1} b_{0} \varepsilon^{1 / m}$.

Очевидно, что $\lim _{\varepsilon \rightarrow 0} b_{0}=1, \lim _{\varepsilon \rightarrow 0} b_{1}=0$. Поскольку $c_{0}$ не зависит от $\varepsilon$, то $c_{0}=\lim _{\varepsilon \rightarrow 0} c_{0}=\lim _{\varepsilon \rightarrow 0} a_{0} \varepsilon^{1 / m}<\infty$. Так как $\Phi_{0}(1, \xi)=-1 / m+\Phi(\xi)$, где $\Phi(\xi)=O\left(1 / \xi^{n}\right)$ при $\xi \rightarrow+\infty$, то

$$
c_{0}=\lim _{\varepsilon \rightarrow 0} a_{0} \varepsilon^{1 / m}=\exp \left(\int_{1}^{0} \frac{\Phi_{0}(1, \xi)}{\xi} d \xi\right) \exp \left(\int_{+\infty}^{1} \frac{\Phi(\xi)}{\xi} d \xi\right)<\infty .
$$

Поскольку $c_{1}$ не зависит от $\varepsilon$, то

$$
c_{1}=\lim _{\varepsilon \rightarrow 0} a_{1} \varepsilon^{1 / m}=\lim _{\varepsilon \rightarrow 0} a_{0} \varepsilon^{1 / m} \int_{1 / \varepsilon}^{0} \frac{\Phi_{1}(1, \xi)}{\xi} \exp \left(\int_{0}^{\xi} \frac{\Phi_{0}(1, \tau)}{\tau} d \tau\right) d \xi=c_{0} I,
$$

где

$$
I=\int_{+\infty}^{0} \frac{\Phi_{1}(1, \xi)}{\xi} \exp \left(\int_{0}^{\xi} \frac{\Phi_{0}(1, \tau)}{\tau} d \tau\right) d \xi
$$


Аналогично, $c_{1}^{x}=c_{0}^{x} I^{x}$, где $c_{0}^{x}, I^{x}$ такие же, как $c_{0}, I$, но соответствуют векторному полю $V^{x}$. Отсюда

$$
\left(\Delta^{x}\right)^{-1} \circ \Delta(\bar{z})=E_{0} \bar{z}\left(1+E_{1} \bar{z}+o(\bar{z})\right),
$$

где с учетом (1.1)

$$
\begin{gathered}
E_{0}=c_{0}\left(c_{0}^{x}\right)^{-1}, \quad E_{1}=c_{1}-c_{0}\left(c_{0}^{x}\right)^{-1} c_{1}^{x}=c_{0} \hat{I}, \\
\hat{I}=I-I_{+\infty}^{x}=\int_{0}^{0} \frac{\Phi_{1}(1, \xi)}{\xi} \exp \left(\int_{0}^{\xi} \frac{\Phi_{0}(1, \tau)}{\tau} d \tau\right) d \xi \\
=\int_{+\infty}^{0} \frac{\Phi_{1}(1, \xi)}{\xi} \exp \left(\int_{0}^{\xi} \frac{\Phi_{0}(1, \tau)}{\tau} d \tau\right) d \xi \\
\quad-\int_{+\infty}^{0} \frac{\Phi_{1}(1,-\xi)}{\xi} \exp \left(\int_{0}^{\xi} \frac{\Phi_{0}^{x}(1, \tau)}{\tau} d \tau\right) d \xi \\
=\int_{+\infty}^{-\infty} \frac{\Phi_{1}(1, \xi)}{\xi} \exp \left(\int_{0}^{\xi} \frac{\Phi_{0}(1, \tau)}{\tau} d \tau\right) d \xi .
\end{gathered}
$$

Аналогично,

$$
\left(\Delta^{y}\right)^{-1} \circ \Delta^{x y}(\bar{z})=E_{0}^{y} \bar{z}\left(1+E_{1}^{y} \bar{z}+o(\bar{z})\right),
$$

где

$$
\begin{gathered}
E_{0}^{y}=c_{0}^{x y}\left(c_{0}^{y}\right)^{-1}, \quad E_{1}^{y}=c_{0}^{x y} \hat{I}^{y}, \\
\hat{I}^{y}=-\int_{+\infty}^{-\infty} \frac{\Phi_{1}^{y}(1, \xi)}{\xi} \exp \left(\int_{0}^{\xi} \frac{\Phi_{0}^{y}(1, \tau)}{\tau} d \tau\right) d \xi .
\end{gathered}
$$

Из (1.1) и (1.3) следует, что $\hat{I}^{y}=\hat{I}$.

Таким образом, преобразование монодромии имеет асимптотику

$$
\hat{\Delta}(\bar{z})=\left(\Delta^{y}\right)^{-1} \circ \Delta^{x y} \circ\left(\Delta^{x}\right)^{-1} \circ \Delta(\bar{z})=E_{0}^{y} E_{0} \bar{z}\left(1+\left(E_{1}+E_{1}^{y} E_{0}\right) \bar{z}+o(\bar{z})\right),
$$

где

$$
E_{0}^{y} E_{0}=c_{0}^{x y}\left(c_{0}^{y}\right)^{-1} c_{0}\left(c_{0}^{x}\right)^{-1}=1,
$$

так как согласно (1.3) при четном $m$ имеем $c_{0}=c_{0}^{y}, c_{0}^{x y}=c_{0}^{x}$. Отсюда

$$
C_{2}=E_{1}+E_{1}^{y} E_{0}=c_{0} \hat{I}+c_{0} c_{0}^{x y}\left(c_{0}^{x}\right)^{-1} \hat{I}^{y}=2 c_{0} \hat{I},
$$

а значит, уравнение $C_{2}=0$ эквивалентно уравнению $-\hat{I}=0$, которое совпадает с (0.4). Теорема доказана.

ДокАЗАТЕЛЬСтво ПРЕДЛОжЕНИЯ 0.2. Ребро диаграммы Ньютона Г имеет показатель $\alpha=m / n$, где $m / n$ - несократимая дробь. Оно лежит на прямой $n i+m j=n m N$, где $N$ - четное число, $N \geqslant 2$, и содержит $N+1$ целочисленных точек. 
Носители многочленов $X_{1}, Y_{1}$ лежат на прямой $\ell_{1}=\{n i+m j=n m N+1\}$. Покажем, что максимально возможный носитель этих многочленов содержит по крайней мере $N$ целочисленных точек. Предположим, что он состоит из $s$ подряд идущих целочисленных точек, где $s \leqslant N-1$. Соединим крайние точки отрезком $Q$. Проекция отрезка $Q$ на ось абсцисс имеет длину $s_{1}=(s-1) m \leqslant$ $(N-2) m$. Проекция же отрезка $Q_{1}$, соединяющего точки пересечения $\ell_{1}$ с осями координат, имеет длину $s_{2}=m N+1 / n$. Имеем $s_{2}-s_{1} \geqslant 2 m+1 / n$. Это значит, что на $Q_{1}$ имеется по крайней мере еще одна целочисленная точка.

Пусть для определенности $F_{0}(x, y)>0$. Учитывая четность $m$, из уравнения $n i+m j=n m N+1$ получаем, что для точек $(i, j)$ носителя $X_{1}, Y_{1}$ все $i$ нечетны, a $j$ чередуются по четности. Поэтому можно выбрать $\left(X_{1}, Y_{1}\right)=x^{i} y^{j}(n, m)$, где $j$ нечетно. Поскольку точка пересечения $\ell_{1}$ с $\{i=0\}$ имеет координату $j_{0}=n N+1 / m$, где $1 / m \leqslant 1 / 2$, то $j \leqslant N n-1$. Так как $\Phi_{1}(x, y)=n x^{i} y^{j} / F_{0}(x, y)$, левая часть (0.4) равна

$$
\text { v.p. } \int_{-\infty}^{+\infty} \frac{n \xi^{j-1}}{F_{0}(1, \xi)} \exp \left(\int_{0}^{\xi} \frac{X_{0}(1, \tau)}{\tau F_{0}(1, \tau)} d \tau\right) d \xi .
$$

Поскольку

$$
\frac{X_{0}(1, \tau)}{\tau F_{0}(1, \tau)}=-\frac{1}{m \tau}+\Psi(\tau),
$$

где $\Psi(\tau)=O\left(1 / \tau^{2}\right)$ при $\tau \rightarrow \infty$, то

$$
\int_{1}^{\xi} \frac{X_{0}(1, \tau)}{\tau F_{0}(1, \tau)} d \tau=-\frac{1}{m} \ln |\xi|+r(\xi),
$$

где $r(\xi)$ ограничена при $\xi \rightarrow \infty$. Поэтому левая часть $(0.4)$ с точностью до умножения на положительное число равна

$$
\int_{-\infty}^{+\infty} \frac{\xi^{j-1} e^{r(\xi)}}{F_{0}(1, \xi)|\xi|^{1 / m}} d \xi
$$

Поскольку степень $F_{0}(1, \xi)$ равна $n N$, то в силу неравенства $N n-j \geqslant 1$ получаем, что этот интеграл сходящийся и положительный.

\section{§2. Случай двух ребер}

В настоящем параграфе докажем теорему 0.2 .

2.1. Раздутие особенности. Пусть диаграмма Ньютона Г векторного поля $V$ состоит из двух ребер $\ell$ и $\tilde{\ell}$ с показателями $\alpha=m / n$ и $\tilde{\alpha}=\tilde{m} / \tilde{n}$ (несократимые дроби), $\alpha<\tilde{\alpha}$, а также выполняются другие условия теоремы 0.2 .

В положительном квадранте $x>0, y>0$ рассмотрим замену переменных

$$
x=w z^{n}, \quad y=z^{m} .
$$

Пусть $\delta, \varepsilon_{1}, \varepsilon_{2}-$ малые положительные числа, $d=\widetilde{m} n-m \tilde{n}$. Образом прямоугольника $P_{\ell}=\left\{(z, w): 0 \leqslant w \leqslant \delta^{-d}, 0 \leqslant z \leqslant \varepsilon_{1}\right\}$ при отображении (2.1) является криволинейный сектор $S_{\ell}$ с вершиной в начале координат, примыкающий к оси $y$. 
В положительном квадранте $x>0, y>0$ рассмотрим также замену переменных

$$
x=\tilde{z}^{\tilde{n}}, \quad y=\tilde{z}^{\widetilde{m}} \widetilde{w} .
$$

Образом прямоугольника $P_{\tilde{\ell}}=\left\{(\tilde{z}, \widetilde{w}): 0 \leqslant \widetilde{w} \leqslant \varepsilon^{-d}, 0 \leqslant \tilde{z} \leqslant \varepsilon_{2}\right\}$ при отображении (2.2) является криволинейный сектор $S_{\tilde{\ell}}$ с вершиной в начале координат, примыкающий к оси $x$.

Пусть $c$ - общая вершина ребер $\ell$ и $\tilde{\ell}$. В малой окрестности нуля между секторами $S_{\ell}$ и $S_{\tilde{\ell}}$ имеется “зазор" в виде криволинейного сектора $S_{c}$. Замена переменных

$$
x=u^{n} v^{\tilde{n}}, \quad y=u^{m} v^{\tilde{m}}
$$

отображает прямоугольник $P_{c}=\left\{0 \leqslant u \leqslant \varepsilon^{\tilde{n}}, 0 \leqslant v \leqslant \delta^{m}\right\}$ в сектор $S_{c}$ (рис. 3 ). Более подробно метод раздутия особенностей, связанный с диаграммой Ньютона, описан в [11].
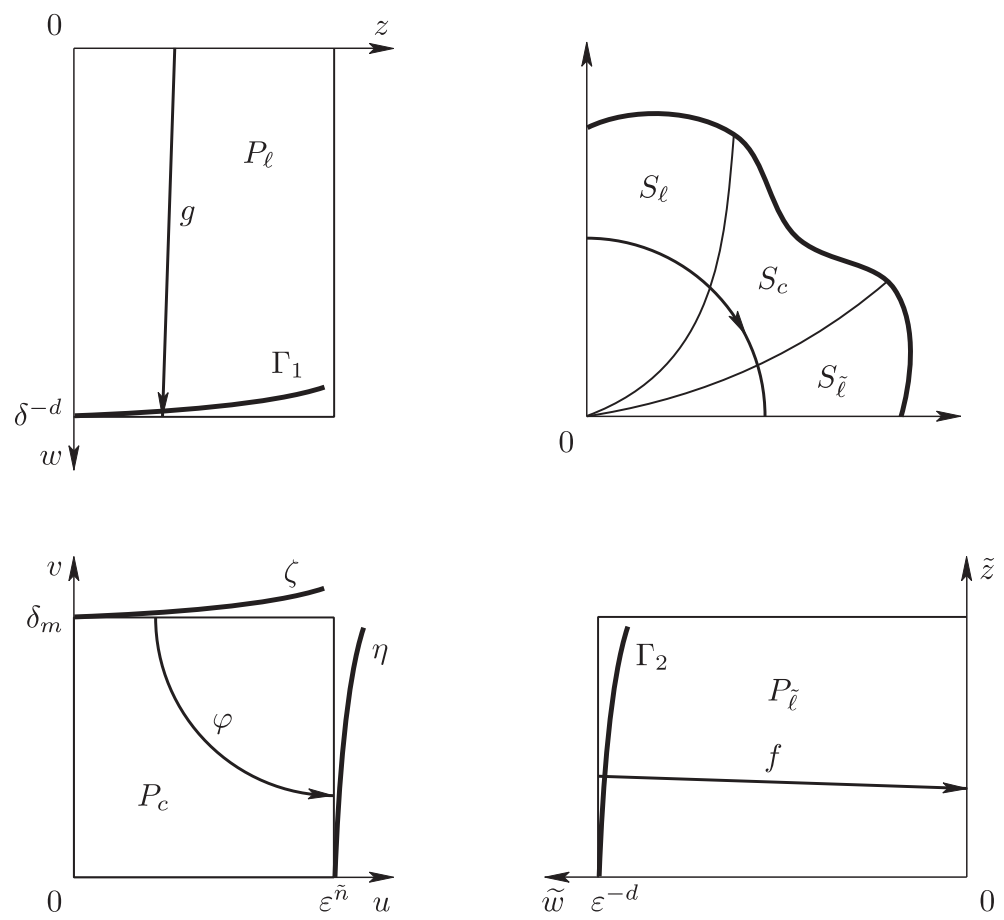

Рис. 3. Раздутие особенности в случае двух ребер

Для того чтобы исследовать исходное векторное поле $V$ в любом другом квадранте, кроме первого, его отражают аналогично случаю одного ребра в первый квадрант. Все обозначения для отраженного векторного поля будут такие же, как для векторного поля $V$, но с соответствующим индексом вверху. 


\section{2. Отображение соответствия в окрестности вырожденного сед-} ла. Напомним, что в секторе $S_{c}$ производится степенная замена (2.3) с матрицей показателей $C_{c}=\left(\begin{array}{cc}n & \stackrel{n}{n} \\ m & \stackrel{m}{m}\end{array}\right)$. Набор собственных значений матрицы линейной части векторного поля $V^{c}$, полученного после этой замены и умножения на некоторые степени $u$ и $v$, в особой точке $(u, v)=(0,0)$ получается из векторного коэффициента $(a, b)$ вершины $c$ с помощью преобразования $C_{c}^{-1}[5]$. Тем самым векторное поле $V^{c}$ после умножения на $-d$ задает систему

$$
\dot{u}=u\left(\lambda_{1}+a^{1}(u, v)\right), \quad \dot{v}=-v\left(\lambda_{2}+a^{2}(u, v)\right),
$$

где $\lambda_{1}=\tilde{n} b-\tilde{m} a, \lambda_{2}=n b-m a, a^{1}(0,0)=a^{2}(0,0)=0 . \quad$ Преобразования носителей при степенных преобразованиях описаны в [5].

Запишем систему (2.4) в виде

$$
\dot{u}=u \sum_{k=0}^{\infty} a_{k}(v) u^{k}, \quad \dot{v}=-v\left(\lambda+\sum_{k=0}^{\infty} b_{k}(v) u^{k}\right)
$$

где $\lambda=\lambda_{2}=n b-m a, a_{0}(0)=\lambda_{1}=\tilde{n} b-\tilde{m} a=0$. Предположим, что $a_{1}(0)=$ $0, \ldots, a_{\nu-1}(0)=0, a_{\nu}(0)=a_{\nu_{0}} \neq 0$. Согласно критерию монодромности [12] величина $\nu$ является четным числом и $\lambda a_{\nu_{0}}>0$. Положим $a=\lambda /\left(\nu a_{\nu_{0}}\right)$. Как показано в [13], с помощью аналитической замены переменных вида

$$
u=\zeta\left(1+\eta r_{0}(\eta)+\eta^{2} r_{1}(\eta) \zeta+O\left(\zeta^{2}\right)\right), \quad v=\eta\left(1+\eta \tilde{r}_{0}(\eta)+\eta^{2} \tilde{r}_{1}(\eta) \zeta+O\left(\zeta^{2}\right)\right)
$$

и деления системы (2.5) на ненулевую аналитическую функцию можно привести данную систему к виду

$$
\dot{\zeta}=\zeta^{\nu+1}, \quad \dot{\eta}=-\eta\left(\nu a+\zeta^{\nu} b(\zeta)+\eta \zeta^{2} G^{*}(\zeta, \eta)\right)
$$

Здесь $r_{i}, \tilde{r}_{i}, b$ - аналитические функции, $G^{*}$ ограничена в нуле.

Пусть $L_{1}=\left\{\eta=\delta^{m}\right\}, L_{2}=\left\{\zeta=\varepsilon^{\tilde{n}}\right\}$, и пусть $\varphi: L_{1} \rightarrow L_{2}$ - отображение соответствия вдоль траекторий системы (2.7), определенное в окрестности нуля на $L_{1}$. Пусть

$$
f_{0}(x)= \begin{cases}e^{-1 / x} & \text { при } x>0 \\ 0 & \text { при } x=0 .\end{cases}
$$

ЛЕмма 2.1 [13]. Имеем $\eta=\varphi(\zeta)=f_{0} \circ t(\zeta)$, где $t(\zeta)=\frac{\zeta^{\nu}}{a}(1+o(\zeta))$.

Непосредственно доказываются следующие леммы.

Лемма 2.2 [2]. Пусть $\hat{f}(\eta)=R_{0} \eta\left(1+R_{1} \eta+o(\eta)\right)$. Тогда

$$
F(\zeta)=f_{0}^{-1} \circ \hat{f} \circ f_{0}(\zeta)=\zeta\left(1+\ln R_{0} \zeta+o(\zeta)\right)
$$

Лемма 2.3. Пустъ $h(x)=\frac{x^{\nu}}{a}(1+o(x)), F(\zeta)=\zeta\left(1+\ln R_{0} \zeta+o(\zeta)\right)$. Тогда $h^{-1} \circ F \circ h(x)=x(1+o(x))$. 
2.3. Отображение соответствия в прямоугольнике, соответствующем ребру. Координаты $(u, v)$ в прямоугольнике $P_{c}$ связаны с координатами $(z, w)$ в прямоугольнике $P_{\ell}$ формулами

$$
z=u v^{\widetilde{m} / m}, \quad w=v^{-d / m} .
$$

Поэтому образ $\Gamma_{1}$ трансверсали $L_{1}=\left\{\eta=\delta^{m}\right\}$ при отображениях (2.6), (2.9) имеет параметризацию

$$
\begin{aligned}
& z=\zeta \delta^{\widetilde{m}}\left(1+o^{\delta}(1)+\delta^{2 m} r_{1}(\delta) \zeta+o(\zeta)\right), \\
& w=\delta^{-d}\left(1+o^{\delta}(1)+\delta^{2 m} \tilde{r}_{1}(\delta) \zeta+o(\zeta)\right),
\end{aligned}
$$

$d=\widetilde{m} n-m \tilde{n}, r_{1}(\delta)$ и $\tilde{r}_{1}(\delta)$ ограничены в нуле. Здесь и везде ниже через $o^{\delta}(1)$ будем обозначать бесконечно малую при $\delta \rightarrow 0$.

Рассмотрим отображение соответствия $\zeta=g(\rho)$ в прямоугольнике $P_{\ell}$, которое отображает сторону $w=0$ в $\Gamma_{1}$, здесь $\rho=z=y^{1 / m}$.

Лемма 2.4. Имеем $\zeta=g(\rho)=g_{0} \rho+g_{1} \rho^{2}+o\left(\rho^{2}\right)$, где

$$
g_{0}=\exp \mid \overline{\int_{0}^{+\infty} \frac{\Psi_{0}(\xi, 1)}{\xi} d \xi}, \quad \frac{g_{1}}{g_{0}}=\int_{0}^{+\infty} \frac{\Psi_{1}(\xi, 1)}{\xi} \exp \left(\int_{0}^{\xi} \frac{\Psi_{0}(\tau, 1)}{\tau} d \tau\right) d \xi
$$

ДокАЗАТЕЛЬство. Векторное поле, полученное после замены (2.1) и определенное в прямоугольнике $P_{\ell}$, задает уравнение

$$
\frac{d z}{d w}=\frac{z}{w}\left(\Psi_{0}(w, 1)+\Psi_{1}(w, 1) z+O\left(z^{2}\right)\right) .
$$

Из условия 1) теоремы 0.2 и из того, что диаграмма Ньютона Г имеет вершину на оси ординат, следует, что данное векторное поле, а также аналогичные векторные поля, соответствующие $V^{x}, V^{y}$ и $V^{x y}$, не имеют особых точек при $z=0$ (многочлены $F_{0}( \pm w, \pm 1)$ не имеют вещественных корней).

Пусть $Z(w, \rho)$ - решение уравнения (2.11), удовлетворяющее условию $\left.Z\right|_{w=0}=\rho$. Тогда

$$
Z(w, \rho)=\rho C_{0}(w)+\rho^{2} C_{1}(w)+o\left(\rho^{2}\right)
$$

где

$$
\begin{gathered}
C_{0}(w)=\exp \int_{0}^{w} \frac{\Psi_{0}(\xi, 1)}{\xi} d \xi \\
C_{1}(w)=C_{0}(w) \int_{0}^{w} \frac{\Psi_{1}(\xi, 1)}{\xi} \exp \left(\int_{0}^{\xi} \frac{\Psi_{0}(\tau, 1)}{\tau} d \tau\right) d \xi .
\end{gathered}
$$

Отображение соответствия $\zeta=g(\rho)$ является решением уравнения $Z(w(\zeta), \rho)=$ $z(\zeta)$, где $z(\zeta), w(\zeta)$ определены в $(2.10)$. Перепишем это уравнение в виде

$$
\rho C_{0}(w(\zeta))+\rho^{2} C_{1}(w(\zeta))+o\left(\rho^{2}\right)=\delta^{\widetilde{m}}\left(1+o^{\delta}(1)\right) \zeta+\delta^{2 m+\widetilde{m}} r_{1}(\delta) \zeta^{2}+o\left(\zeta^{2}\right) .
$$

Найдем $\zeta=g(\rho)$ в виде

$$
\zeta=g(\rho)=g_{0} \rho+g_{1} \rho^{2}+o\left(\rho^{2}\right) .
$$


Разложим $C_{0}(w(\zeta))$ и $C_{1}(w(\zeta))$ по степеням $\zeta$ :

$$
\begin{aligned}
C_{0}(w(\zeta))= & C_{0}\left(\delta^{-d}\left(1+o^{\delta}(1)\right)+\delta^{2 m-d} \tilde{r}_{1}(\delta) \zeta+o(\zeta)\right) \\
= & C_{0}\left(\delta^{-d}\left(1+o^{\delta}(1)\right)\right)+C_{0}^{\prime}\left(\delta^{-d}\left(1+o^{\delta}(1)\right)\right) \delta^{2 m-d} \tilde{r}_{1}(\delta) \zeta+o(\zeta) \\
= & C_{0}\left(\delta^{-d}\left(1+o^{\delta}(1)\right)\right)+\frac{\Psi_{0}\left(\delta^{-d}\left(1+o^{\delta}(1)\right), 1\right)}{\delta^{-d}\left(1+o^{\delta}(1)\right)} \\
& \quad \times C_{0}\left(\delta^{-d}\left(1+o^{\delta}(1)\right)\right) \delta^{2 m-d} \tilde{r}_{1}(\delta) \zeta+o(\zeta) \\
= & C_{0}\left(\delta^{-d}\left(1+o^{\delta}(1)\right)\right)+\Psi_{0}\left(\delta^{-d}\left(1+o^{\delta}(1)\right), 1\right) \\
& \times C_{0}\left(\delta^{-d}\left(1+o^{\delta}(1)\right)\right)\left(1+o^{\delta}(1)\right) \delta^{2 m} \tilde{r}_{1}(\delta) \zeta+o(\zeta) \\
C_{1}(w(\zeta))= & C_{1}\left(\delta^{-d}\left(1+o^{\delta}(1)\right)+O(\zeta)\right)=C_{1}\left(\delta^{-d}\left(1+o^{\delta}(1)\right)\right)+O(\zeta) .
\end{aligned}
$$

Подставим (2.13) в (2.14) и (2.15), а затем в (2.12). Получим

$$
\begin{aligned}
C_{0}\left(\delta^{-d}\left(1+o^{\delta}(1)\right)\right) \rho+\Psi_{0}\left(\delta^{-d}\left(1+o^{\delta}(1)\right), 1\right)\left(1+o^{\delta}(1)\right) \\
\quad \times C_{0}\left(\delta^{-d}\left(1+o^{\delta}(1)\right)\right) \delta^{2 m} \tilde{r}_{1}(\delta) g_{0} \rho^{2}+C_{1}\left(\delta^{-d}\left(1+o^{\delta}(1)\right)\right) \rho^{2}+o\left(\rho^{2}\right) \\
=\delta^{\widetilde{m}}\left(1+o^{\delta}(1)\right) g_{0} \rho+\rho^{2}\left[\delta^{\widetilde{m}}\left(1+o^{\delta}(1)\right) g_{1}+\delta^{2 m+\widetilde{m}} r_{1}(\delta) g_{0}^{2}\right]+o\left(\rho^{2}\right) .
\end{aligned}
$$

Приравняем коэффициенты при $\rho$ и $\rho^{2}$ :

$$
\begin{gathered}
C_{0}\left(\delta^{-d}\left(1+o^{\delta}(1)\right)\right)=\delta^{\widetilde{m}}\left(1+o^{\delta}(1)\right) g_{0} \\
\Psi_{0}\left(\delta^{-d}\left(1+o^{\delta}(1)\right), 1\right)\left(1+o^{\delta}(1)\right) C_{0}\left(\delta^{-d}\left(1+o^{\delta}(1)\right)\right) \delta^{2 m} \tilde{r}_{1}(\delta) g_{0} \\
+C_{1}\left(\delta^{-d}\left(1+o^{\delta}(1)\right)\right)=\delta^{\widetilde{m}}\left(1+o^{\delta}(1)\right) g_{1}+\delta^{2 m+\widetilde{m}} r_{1}(\delta) g_{0}^{2} .
\end{gathered}
$$

Отсюда получаем

$$
\begin{gathered}
g_{0}=\delta^{-\widetilde{m}} C_{0}\left(\delta^{-d}\left(1+o^{\delta}(1)\right)\right)\left(1+o^{\delta}(1)\right) \\
g_{1}=\delta^{-\widetilde{m}} C_{0}\left(\delta^{-d}\left(1+o^{\delta}(1)\right)\right)\left(1+o^{\delta}(1)\right) \Psi_{0}\left(\delta^{-d}\left(1+o^{\delta}(1)\right), 1\right) \delta^{2 m} \tilde{r}_{1}(\delta) g_{0} \\
+\delta^{-\widetilde{m}} C_{1}\left(\delta^{-d}\left(1+o^{\delta}(1)\right)\right)\left(1+o^{\delta}(1)\right)-\delta^{2 m} r_{1}(\delta) g_{0}^{2}\left(1+o^{\delta}(1)\right)
\end{gathered}
$$

Поскольку отображение $f_{0} \circ t \circ g$ не зависит от $\delta$ и коэффициенты разложения $t$ не зависят от $\delta$, то и коэффициенты разложения $g$ не зависят от $\delta$ (имеются в виду только контролируемые коэффициенты). Таким образом, $g_{0}=\lim _{\delta \rightarrow 0} g_{0}, g_{1}=\lim _{\delta \rightarrow 0} g_{1}$. Вычислим эти пределы. Так как

$$
\frac{\Psi_{0}(\xi, 1)}{\xi}=-\frac{Y_{0}(\xi, 1)}{\xi F_{0}(\xi, 1)}=-\frac{b}{n b-m a} \frac{1}{\xi}+\Psi(\xi),
$$

где $\Psi(\xi)=O\left(1 / \xi^{2}\right)$ при $\xi \rightarrow \infty$, то

$$
\begin{aligned}
\int_{0}^{\delta^{-d}\left(1+o^{\delta}(1)\right)} & \frac{\Psi_{0}(\xi, 1)}{\xi} d \xi \\
= & \int_{0}^{1} \frac{\Psi_{0}(\xi, 1)}{\xi} d \xi+\int_{1}^{\delta^{-d}\left(1+o^{\delta}(1)\right)}\left(-\frac{b}{n b-m a} \frac{1}{\xi}+\Psi(\xi)\right) d \xi \\
= & \int_{0}^{1} \frac{\Psi_{0}(\xi, 1)}{\xi} d \xi+\frac{b d}{n b-m a} \ln \delta+\int_{1}^{\delta^{-d}\left(1+o^{\delta}(1)\right)} \Psi(\xi) d \xi+o^{\delta}(1) .
\end{aligned}
$$


Учитывая, что $\tilde{n} b-\widetilde{m} a=0$, получаем $\frac{b d}{n b-m a}=\widetilde{m}$. Отсюда при $\delta \rightarrow 0$ имеем

$$
\begin{aligned}
& \delta^{-\widetilde{m}} C_{0}\left(\delta^{-d}\left(1+o^{\delta}(1)\right)\right)=\delta^{-\widetilde{m}} \exp \left(\int_{0}^{\delta^{-d}\left(1+o^{\delta}(1)\right)} \frac{\Psi_{0}(\xi, 1)}{\xi} d \xi\right) \\
& \longrightarrow \exp \left(\int_{0}^{1} \frac{\Psi_{0}(\xi, 1)}{\xi} d \xi\right) \exp \left(\int_{1}^{+\infty} \Psi(\xi) d \xi\right)=g_{0}=\exp \left(\mid \overline{\int_{0}^{+\infty} \frac{\Psi_{0}(\xi, 1)}{\xi} d \xi}\right) .
\end{aligned}
$$

Теперь, учитывая ограниченность $g_{0}, \quad r_{1}, \quad \tilde{r}_{1}, \quad \delta^{-\widetilde{m}} C_{0}\left(\delta^{-d}\left(1+o^{\delta}(1)\right)\right)$, $\Psi_{0}\left(\delta^{-d}\left(1+o^{\delta}(1)\right), 1\right)$, при $\delta \rightarrow 0$ получаем, что первое и третье слагаемые в соотношении (2.16) стремятся к нулю.

Таким образом,

$$
\begin{aligned}
g_{1} & =\lim _{\delta \rightarrow 0} \delta^{-\widetilde{m}} C_{0}\left(\delta^{-d}\left(1+o^{\delta}(1)\right)\right) \int_{0}^{\delta^{-d}\left(1+o^{\delta}(1)\right)} \frac{\Psi_{1}(\xi, 1)}{\xi} \exp \left(\int_{0}^{\xi} \frac{\Psi_{0}(\tau, 1)}{\tau} d \tau\right) d \xi \\
& =g_{0} \int_{0}^{+\infty} \frac{\Psi_{1}(\xi, 1)}{\xi} \exp \left(\int_{0}^{\xi} \frac{\Psi_{0}(\tau, 1)}{\tau} d \tau\right) d \xi .
\end{aligned}
$$

Итак, $g(\rho)=g_{0} \rho\left(1+\hat{g}_{1} \rho\right)$, где

$$
\hat{g}_{1}=\frac{g_{1}}{g_{0}}=\int_{0}^{+\infty} \frac{\Psi_{1}(\xi, 1)}{\xi} \exp \left(\int_{0}^{\xi} \frac{\Psi_{0}(\tau, 1)}{\tau} d \tau\right) d \xi .
$$

Лемма доказана.

2.4. Асимптотика преобразования монодромии. Рассмотрим преобразование монодромии $\hat{\Delta}$, определенное на положительной полуоси $y$ с параметром $\rho=y^{1 / m}$. Оно представляется в виде композиции $\hat{\Delta}=\Delta_{2} \circ \Delta_{1}$, где $\Delta_{1}$ и $\Delta_{2}$ - отображения соответствия для правой и левой полуплоскости.

Отображение $\Delta_{1}$ является композицией шести отображений соответствия:

$$
\Delta_{1}=\left(g^{x}\right)^{-1} \circ\left(\varphi^{x}\right)^{-1} \circ\left(f^{x}\right)^{-1} \circ f \circ \varphi \circ g,
$$

где $g^{x}, \varphi^{x}$ аналогичны $g, \varphi$, но соответствуют векторному полю $V^{x} ; f, f^{x}-$ отображения соответствия в прямоугольнике $P_{\tilde{\ell}}$ для $V, V^{x}$ соответственно; $f, f^{x}$ имеют линейные главные члены асимптотики, поскольку векторные поля, полученные после замены $(2.2)$ и определенные в $P_{\tilde{\ell}}$, не имеют особых точек при $\tilde{z}=0$. Заметим, что для всех векторных полей $V, V^{x}, V^{y}, V^{x y}$ величина $a=\lambda /\left(\nu a_{\nu_{0}}\right)$ одна и та же, поэтому отображение соответствия $\varphi(\zeta)$, описанное в лемме 2.1, задается одной и той же формулой для всех четырех векторных полей. Отсюда и из лемм 2.1-2.3 получаем, что $\Delta_{1}=\left(g^{x}\right)^{-1} \circ g, \Delta_{2}=\left(g^{y}\right)^{-1} \circ g^{x y}$ с точностью до $о\left(\rho^{2}\right)$. Если

$$
g(\rho)=g_{0} \rho\left(1+\hat{g}_{1} \rho+o(\rho)\right), \quad g^{x}(\rho)=g_{0}^{x} \rho\left(1+\hat{g}_{1}^{x} \rho+o(\rho)\right),
$$

то имеем

$$
\Delta_{1}(\rho)=\frac{g_{0}}{g_{0}^{x}} \rho\left(1+\left(\hat{g}_{1}-\frac{g_{0}}{g_{0}^{x}} \hat{g}_{1}^{x}\right) \rho+o(\rho)\right) .
$$


Аналогично,

$$
\Delta_{2}(\rho)=\frac{g_{0}^{x y}}{g_{0}^{y}} \rho\left(1+\left(\hat{g}_{1}^{x y}-\frac{g_{0}^{x y}}{g_{0}^{y}} \hat{g}_{1}^{y}\right) \rho+o(\rho)\right) .
$$

И наконец, $\Delta(\rho)=C_{1} \rho\left(1+\widehat{C}_{2} \rho+o(\rho)\right)$, где

$$
C_{1}=\frac{g_{0}^{x y} g_{0}}{g_{0}^{y} g_{0}^{x}}, \quad \widehat{C}_{2}=\frac{g_{0}}{g_{0}^{x}} \hat{g}_{1}^{x y}-\frac{g_{0}}{g_{0}^{x}} \frac{g_{0}^{x y}}{g_{0}^{y}} \hat{g}_{1}^{y}+\hat{g}_{1}-\frac{g_{0}}{g_{0}^{x}} \hat{g}_{1}^{x} .
$$

Рассмотрим два случая:

1) если $m$ нечетно, то согласно (1.1), (1.2) имеем

$$
g_{0}=g_{0}^{x}, \quad g_{0}^{x y}=g_{0}^{y}, \quad g_{1}^{x}=-g_{1} \quad g_{1}^{x y}=-g_{1}^{y},
$$

отсюда получаем

$$
\begin{gathered}
C_{1}=1, \\
C_{2}=\widehat{C}_{2}=\hat{g}_{1}^{x y}-\hat{g}_{1}^{y}+\hat{g}_{1}-\hat{g}_{1}^{x}=2 \hat{g}_{1}-2 \hat{g}_{1}^{y} \\
=2 \int_{0}^{+\infty} \frac{\Psi_{1}(\xi, 1)}{\xi} \exp \left(\int_{0}^{\xi} \frac{\Psi_{0}(\tau, 1)}{\tau} d \tau\right) d \xi \\
-2 \int_{0}^{+\infty} \frac{\Psi_{1}(-\xi, 1)}{\xi} \exp \left(\int_{0}^{\xi} \frac{\Psi_{0}(-\tau, 1)}{\tau} d \tau\right) d \xi \\
=2 \int_{-\infty}^{+\infty} \frac{\Psi_{1}(\xi, 1)}{\xi} \exp \left(\int_{0}^{\xi} \frac{\Psi_{0}(\tau, 1)}{\tau} d \tau\right) d \xi
\end{gathered}
$$

2) если $m$ четно, то согласно (1.1), (1.3) имеем $g_{0}=g_{0}^{y}, g_{0}^{x y}=g_{0}^{x}$, отсюда получаем

$$
C_{1}=1, \quad C_{2}=\widehat{C}_{2}=\hat{g}_{1}-\hat{g}_{1}^{y}+\frac{g_{0}}{g_{0}^{x}}\left(\hat{g}_{1}^{x y}-\hat{g}_{1}^{x}\right)
$$

Далее, так как главные части $\Psi_{0}(\xi, 1) / \xi$ и $\Psi_{0}(\xi,-1) / \xi$ в бесконечности совпадают, то

$$
\begin{aligned}
\frac{g_{0}}{g_{0}^{x}} & =\exp \left(\mid \overline{\int_{0}^{+\infty} \frac{\Psi_{0}(\xi, 1)}{\xi} d \xi}\right) \exp \left(-\sqrt{\int_{0}^{+\infty} \frac{\Psi_{0}^{x}(\xi, 1)}{\xi} d \xi}\right) \\
& =\exp \left(\int_{0}^{+\infty}\left(\Psi_{0}(\xi, 1)-\Psi_{0}(\xi,-1)\right) \frac{d \xi}{\xi}\right) .
\end{aligned}
$$

Отсюда и из (2.17) имеем

$$
\begin{aligned}
C_{2}= & \int_{-\infty}^{+\infty} \frac{\Psi_{1}(\xi, 1)}{\xi} \exp \left(\int_{0}^{\xi} \frac{\Psi_{0}(\tau, 1)}{\tau} d \tau\right) d \xi-\exp \left(\int_{0}^{+\infty}\left(\Psi_{0}(\xi, 1)-\Psi_{0}(\xi,-1)\right) \frac{d \xi}{\xi}\right) \\
& \times \int_{-\infty}^{+\infty} \frac{\Psi_{1}(\xi,-1)}{\xi} \exp \left(\int_{0}^{\xi} \frac{\Psi_{0}(\tau,-1)}{\tau} d \tau\right) d \xi .
\end{aligned}
$$

Теорема 0.2 доказана. 
ДокАЗАТЕЛЬСТво СЛЕДСТВИЯ 0.1. Умножим (2.18) на

$$
g_{0}^{-1}=\exp \left(-\sqrt{\int_{0}^{+\infty} \frac{\Psi_{0}(\xi, 1)}{\xi} d \xi}\right)=\exp \left(\sqrt{\int_{+\infty}^{0} \frac{\Psi_{0}(\xi, 1)}{\xi} d \xi}\right)
$$

и внесем в первый большой интеграл из (2.18). Аналогично, $\left(g_{0}^{x}\right)^{-1}$ внесем во второй большой интеграл из (2.18). После приравнивания $C_{2}$ нулю получим уравнение $(0.5)$.

ДоКАЗАТЕЛЬСТВо ПРЕДЛОЖЕНИЯ 0.3. Согласно критерию монодромности [12] проекции обоих ребер диаграммы Ньютона $Г$ на координатные оси являются четными числами. Отсюда $k_{0}$ - четное число (ребро $\ell$ диаграммы Ньютона Г лежит на прямой $n i+m j=k_{0}$ ).

Пусть для определенности $F_{0}(x, y)>0$. В случае четного $m$ получаем из уравнения $n i+m j=k_{0}+1$, что для точек носителей $X_{1}, Y_{1}$ все $i$ нечетны, а четность $j$ чередуется. В случае нечетного $m$ все $j$ четны, а четность $i$ чередуется. В каждом из этих случаев можно выбрать $\left(X_{1}, Y_{1}\right)=x^{i} y^{j}(n, m)$, где $i$ нечетно. Тогда

$$
\frac{\Psi_{1}(\xi, 1)}{\xi}=-m \frac{\xi^{i-1}}{F_{0}(\xi, 1)}, \quad \frac{\Psi_{1}(\xi,-1)}{\xi}=m \frac{\xi^{i-1}}{F_{0}(\xi, 1)} .
$$

При таком выборе получаем, что $C_{2}$ в обоих случаях отрицательно.

\section{Список литературы}

1. В.И. Арнольд, Ю.С. Ильяшенко, "Обыкновенные дифференциальные уравнения", Динамические системь - 1, Итоги науки и техн. Сер. Соврем. пробл. матем. Фундам. направления, 1, ВИНИТИ, М., 1985, 7-149; англ. пер.: V.I. Arnol'd, Yu.S. Il'yashenko, "Ordinary differential equations", Dynamical systems I, Encyclopaedia Math. Sci., Springer-Verlag, Berlin, 1988, 1-148.

2. Ю. С. Ильяшенко, "Мемуар Дюлака "О предельных циклах" и смежные вопросы локальной теории дифференциальных уравнений", УМН, 40:6 (1985), 41-78; англ. пер.: Yu. S. Il'yashenko, "Dulac's memoir "On limit cycles" and related problems of the local theory of differential equations", Russian Math. Surveys, 40:6 (1985), 1-49.

3. Н. Б. Медведева, "Главный член преобразования монодромии монодромной особой точки линеен", Сиб. матем. журн., 33:2 (1992), 116-124; англ. пер.: N. B. Medvedeva, "Principal term of the monodromy transformation of a monodromic singular point is linear", Siberian Math. J., 33:2 (1992), 280-288.

4. Н. Б. Медведева, Е. В. Мазаева, “Достаточное условие фокуса для монодромной особой точки", Тр. ММО, 63, УРСС, М., 2002, 87-114; англ. пер.: N. B. Medvedeva, E. V. Mazaeva, "A sufficient condition for a focus for a monodromic singular point", Trans. Moscow Math. Soc., 2002 (2002), 77-103.

5. Ф.С. Березовская, Н. Б. Медведева, "Асимптотика преобразования монодромии особой точки с фиксированной диаграммой Ньютона", Тр. сем. им. И. Г. Петровского, 15, Изд-во Моск. ун-та, М., 1991, 156-177; англ. пер.: F. S. Berezovskaya, N. B. Medvedeva, "The asymptotics of the return map of a singular point with fixed Newton diagram", J. Soviet Math., 60:6 (1992), 1765-1781.

6. А. С. Воронин, Н. Б. Медведева, "Устойчивость монодромных особых точек с фиксированной диаграммой Ньютона", Вестн. Удмуртского ун-та. Сер. матем., мех., комп. науки, 3 (2009), 34-49. 
7. А. С. Воронин, Н. Б. Медведева, “Асимптотика преобразования монодромии в случае двух четных ребер диаграммы Ньютона", Вестн. ЧелГУ, Сер. 3. Матем., мех., информ., 27(242):14 (2011), 12-26.

8. А. Д. Брюно, Локальный метод нелинейного анализа дифференциальных уравнений, Наука, М., 1979; англ. пер.: А. D. Bruno, Local methods in nonlinear differential equations, Springer Ser. Soviet Math., Springer-Verlag, Berlin, 1989.

9. В.П. Варин, "Отображение последования некоторых полиномиальных систем дифференциальных уравнений”, Матем. сб., 195:7 (2004), 3-20; англ. пер.: V.P. Varin, "Poincaré map for some polynomial systems of differential equations", Sb. Math., 195:7 (2004), 917-934.

10. Ж. Адамар, Задача Коши для линейных уравнений с частными производными гиперболического типа, Наука, М., 1978; пер. с англ.: J. Hadamard, Lectures on Cauchy's problem in linear partial differential equations, Dover, New York, 1953.

11. Н. Б. Медведева, "Об аналитической разрешимости проблемы различения центра и фокуса", Нелинейные аналитические дифференциальные уравнения, Тр. МИАН, 254, Наука, М., 2006, 11-100; англ. пер.: N. B. Medvedeva, "On the analytic solvability of the problem of distinguishing between center and focus", Proc. Steklov Inst. Math., 254 (2006), 7-93.

12. Н.Б. Медведева, "Критерий монодромности особой точки векторного поля на плоскости", Алгебра и анализ, 13:2 (2001), 130-150; англ. пер.: N. B. Medvedeva, "А monodromy criterion for a singular point of a vector field on the plane", St. Petersburg Math. J., 13:2 (2002), 253-268.

13. Н. Б. Медведева, "Проблема различения центра и фокуса в классе ростков с двумя ребрами диаграммы Ньютона", Вестн. ЧелГУ, Сер. 3. Матем., мех., информ., 3:9 (2003), 86-110.

А. С. Воронин (А. S. Voronin)

Челябинский государственный университет

E-mail: neizvest71@mail.ru

Н. Б. Медведева (N. B. Medvedeva)

Челябинский государственный университет

E-mail: medv@csu.ru
Поступило в редакцию 29.03.2012

04.07 .2012 